\title{
Classificação de sistemas dinâmicos pelas superfícies de erros de predição cruzada
}

\author{
André Fonseca \\ Centro de Matemática, Computação e Cognição da Universidade Federal do ABC \\ Rua Arcturus 3 - Bloco Delta - Sala 280 \\ 09606-070 - São Bernardo do Campo - SP - Brazil \\ E-mail: andre.fonseca@ufabc.edu.br. \\ Birajara Soares Machado \\ Instituto do Cérebro, Hospital Israelita Albert Einstein \\ Av. Albert Einstein, 627/701 \\ 05652-900 - São Paulo - SP - Brazil \\ E-mail: birasm@gmail.com.
}

\section{Resumo}

Neste trabalho desenvolvemos um estimador de complexidade para a análise de sinais empíricos não estacionários, baseado no estudo dos erros de predição cruzada. Esses erros são relacionados à mudanças na estrutura de recorrência e na distribuição de probabilidade dos estados. O estimador avalia a componente determinística ou estocástica do mecanismo gerador e seus resultados foram promissores quando comparados com as medidas Lempel-Ziv e expoente de Lyapunov, calculados em sistemas dinâmicos clássicos.

Palavras-chave: sistemas dinâmicos, erro de predição cruzada, determinismo, estacionaridade.

\section{Introdução}

Lorenz, em seus estudos atmosféricos [1], analisou o erro de predição para estados recorrentes ou "análogos". Ele afirmou que a obtenção de previsões "irreais" de um modelo está relacionada com a perda de periodicidade ou de sua estrutura de recorrência. Quando essa estrutura se colapsa, temos o fenômeno aleatório. Diversos autores têm analisado a perda de recorrência e da capacidade de predição nos sinais, como Schreiber, que explorou a característica não estacionária do sistemas dinâmicos [2]. A predição "falha" quando os momentos estatísticos não são constantes no tempo. Desta forma, a perda de recorrência ou a mudança nas distribuições de probabilidade dos estados afetam consideravelmente a acurácia da predição dos modelos escolhidos para análise de sinais empíricos. Para o cenário não estacionário, a classificação de seu mecanismo gerador ainda apresenta incertezas.

\section{Metodologia}

O erro de predição cruzada estima a acurácia da predição de estados de um subconjunto utilizando estados de outro subconjunto, onde esses subconjuntos foram construídos a partir do mesmo sinal. Dividimos uma série temporal com $N$ pontos em $w=N / l$ janelas sucessivas de tamanho $l$ definidas como $W_{i}=\left\{x_{(i-1) l+1}, \ldots, x_{i l}\right\}$. Para cada par $W_{i}, W_{j}$ onde $i, j=1, \ldots, n / l$ obtemos, respectivamente, os vetores $\overrightarrow{x_{k}}=\left(x_{k-m+1}, \ldots, x_{k}\right)$ e $\overrightarrow{y_{k}}=\left(y_{k-m+1}, \ldots, y_{k}\right)$ onde $m$ é a dimensão de imersão e $\tau$ é a defasagem temporal. Para prever $y_{k+1}$ nós usamos o estimador: 


$$
\hat{y}_{k+1}^{x}=\frac{1}{\# S_{\varepsilon}^{\vec{x}}} \sum_{\overrightarrow{x_{p}} \in S_{\varepsilon}^{\vec{x}}} x_{p+1} ; S_{\varepsilon}^{\vec{x}}=\left\{\overrightarrow{x_{p}}:\left\|\overrightarrow{x_{p}}-\overrightarrow{y_{k}}\right\|<\varepsilon\right\},
$$

A matriz ou superfície de erros de predição cruzada é definida como:

$$
\gamma(i, j)=\gamma\left(W_{i}, W_{j}\right)=\sqrt{\frac{1}{l-m} \sum_{k=m}^{l-1}\left(\hat{y}_{k+1}^{x}-y_{k+1}\right)^{2}} .
$$

Dado um sinal $x$, realizamos uma permutação aleatória obtendo o sinal $y$. Calculamos as matrizes $\gamma_{x}(i, j)$ e $\gamma_{y}(i, j)$. O estimador de complexidade proposto é definido como $\bar{\delta}=$ $\left\langle\left|\gamma_{x}(i, j)-\gamma_{y}(i, j)\right|\right\rangle ; i, j=1, \ldots, w$.

\section{Resultados}

Na figura 1 observamos o resultado da medida de complexidade aplicada a sinais do mapa logístico com diferentes regimes.
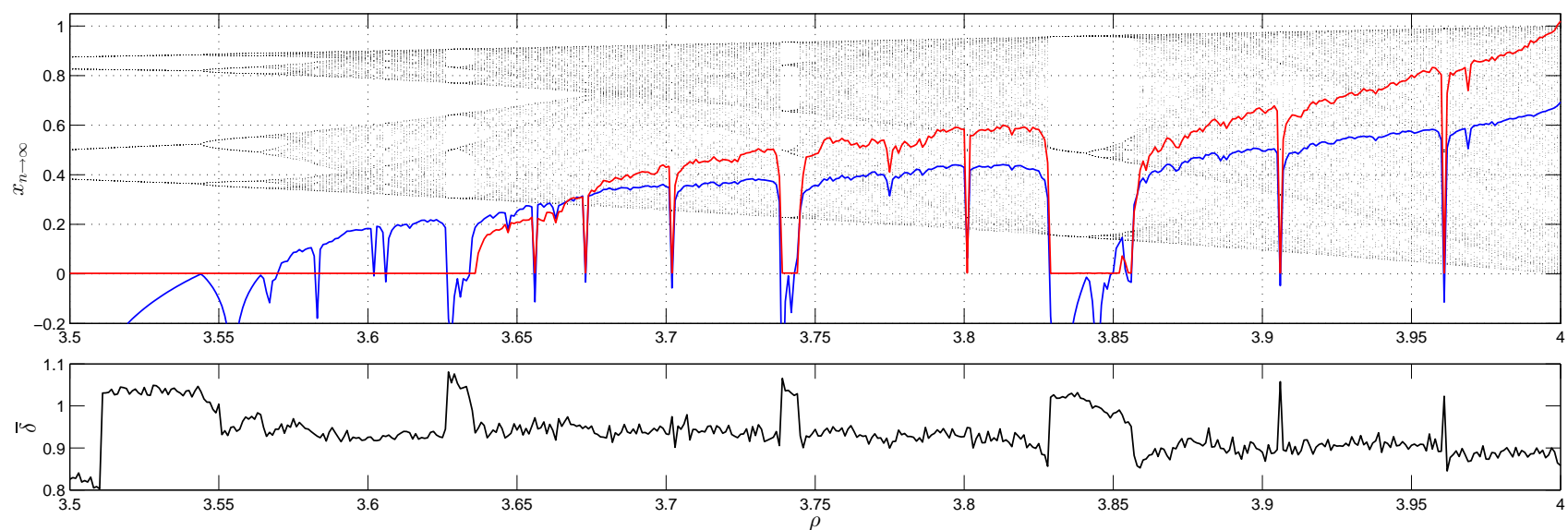

Figura 1: Complexidade Lempel-Ziv (vermelho), expoente de Lyapunov (azul) e $\bar{\delta}$ para o diagrama de bifurcação do mapa logístico.

\section{Conclusão}

O estimador de complexidade proposto apresenta resultados promissores e robustos frente à perturbação dos parâmetros de controle, auxiliando na caracterização do mecanismo gerador de sinais empíricos.

\section{Referências}

[1] Lorenz, E. N. Atmospheric predictability as revealed by naturally occurring analogues. J. Atmos. Sci., 26, 636-646 (1969).

[2] Schreiber, T. Detecting and Analyzing Nonstationarity in a Time Series Using Nonlinear Cross Predictions. Phys. Rev. Lett, 78, 843-846 (1997). 